\title{
Research Progress of Biodegradable Medical Materials
}

\author{
Guojun Shang ${ }^{1, a}$ \\ ${ }^{1}$ School of Material Science and Engineering, Beijing Institute of Technology, Beijing 100081, China; \\ aevangeline1417@163.com
}

Keywords: Biodegradable medical materials; Polymer; Bioceramic materials; Medical applications.

\begin{abstract}
Biodegradable medical materials are widely used in the field of medicine, which mainly include biodegradable polymer materials, biodegradable ceramic materials, biological derivatives, biological hybrid materials etc. In this paper, we discuss the properties and applications of biodegradable medical materials in medical field according to different classification methods.
\end{abstract}

\section{Introduction}

Biodegradable biomedical materials refer to materials which can be placed into the human body and can be broken down or destroyed after a period of time, the implants will decompose or degrade into non-toxic, harmless and small molecules and will be expelled from the human body after the mission completed [1-5]. We need some temporary material as a support or a carrier in medicine, such as fracture fixation, drug controlled release, which requires good biodegradability for the implanted materials during wound healing or drug release process. Biodegradable medical materials have the characteristic of good biocompatibility, biodegradability, non-toxic. So far, the most widely studied biodegradable medical materials include biomedical polymer materials, such as poly hydroxy fatty acid ester; bioceramic materials, such as calcium phosphate ceramics, ceramic apatite; biomedical composite materials, such as polylactic acid, polyglycolic acid etc. Biodegradable materials have been widely used in the field of medical suture, fracture fixation, cancer treatment, family planning, drug delivery system, organ repair and tissue engineering etc. [2, 3].

\section{The classification of biodegradable materials}

There are many kinds of biodegradable materials, of which biodegradable polymer materials and biodegradable ceramic materials are the most currently studied, and other biological derivatives, hybrid materials etc.. In this paper, the research progress of different biodegradable medical materials is discussed [2, 4, 5].

\subsection{Biodegradable polymer materials}

Polymer materials have long been used in the field of medicine and so far have become a relatively mature and a wide variety of biomedical materials. According to the origin, it can be divided into two kinds: natural and synthetic polymer materials and there are two degradation modes: chemical degradation and biodegradation [6].

\subsubsection{Natural biodegradable polymer materials}

Natural biodegradable polymer materials are a kind of semi-artificial synthetic polymeric materials which is obtained by the modification of natural polymers as raw materials. Most of the natural biopolymers can be degraded by enzymes in vivo. The following lists several major natural biodegradable polymer materials and their applications [7-10, 12].

Collagen is a group of animal protein with similar structure and abundant, an important part of human tissue, so it is very suitable for the repair and regeneration of human organs and tissues. It is quite common in clinical medicine, especially in plastic surgery and medical cosmetology. A kind of injectable collagen liquid can be used for subcutaneous implantation to correct a variety of small defects on the skin, the materials degrade and absorb only in the affected region. The amino acid was used as a raw material for repairing tissue by the protease digestion of local white blood cells and macrophages. 
Chitin is a kind of inert polysaccharide that widely existed in insect shells. Compared with catgut, the absorption cycle of chitin fiber is longer, which is conducive to wound healing. While because its strength is not so high, it is still difficult for application. The derivative chitosan can inhibit the absorption of bile salts and other lipid compounds and make them out of body, thereby lowering cholesterol and blood lipid in vivo. Chitosan also has a strong inhibitory effect on tumor, can enhance the effect of lymphocyte for killing cancer cells when it reaches a certain concentration.

Fibrin is a plasma protein, the function is to participate in the coagulation process for stopping bleeding and promoting tissue healing, it has very important applications in the biomedical field. In addition, as a component of natural extracellular matrix, fibrin has better performance of cell signal transduction and interaction. Fibrin gel and polymeric materials are used to construct an in vitro tissue engineering cartilage model, which is linked to the cartilage cells and similar to the cartilage of the body. The degradation of fibrin includes two processes of enzymatic degradation and cell phagocytosis and the degradation products can be completely absorbed.

\subsubsection{Synthetic biodegradable polymer materials}

Generally speaking, the synthesis of synthetic biodegradable polymer materials is mainly by controlling the conditions to produce reproducible materials and design the production method according to the needs to obtain extensive performance to meet the different needs by means of simple physical and chemical modification, so that it has more advantages than the natural ones. Therefore, the synthesis of synthetic biodegradable polymer materials are more widely used in biomedical applications.

Polylactic acid is currently one of the most promising biodegradable polymer. Its initial raw material is mainly starch. The products are carbon dioxide and water after the biodegradation of polylactic acid without any environmental problems. After implanted into human body, the inflammatory reaction with the human body is not obvious because of its slow rate of degradation. We can accelerate its degradation rate by adding some hydrophilic components or reducing the crystallinity. Due to the optical activity of lactic acid, there also has three kinds of poly lactic acid and the strength and degradation cycle of the materials can be adjusted by the different proportions of the three kinds of polylactic acid.

Ortho ester is a special ether compound formed by condensation of the original acid and alcohol with heterogeneous degradation mechanism, which is especially suitable for the preparation of drug release materials. Because of the existence of the acid sensitive raw ester bonds in its main chain, we can control the drug release behavior by adding acid or alkaline excipient. Currently there are mainly three kinds of esters be used in the field of medicin, mostly they are used as drug control release carrier, also they can be used as the treatment materials for burned parts. Because of acid production in the process of degradation in vivo, ortho ester has the property of self catalytic, and the degradation rate is sure to be accelerated.

Polyamino acids and its copolymers are also widely used in biomedical materials owing to the characteristics of good biocompatibility and biodegradability and the degradation products has no toxic effects on the human body. There are many reactive pendant groups on the end of amino acid chain such as amino, carboxyl, which can be used to immobilize biologically active molecules, such as proteins carbohydrate and peptide, improving the interaction between the materials and cells. Since amino acids main contact with body fluids and tissues in the human body, hydrolysis and enzymatic hydrolysis are the most important degradation system.

\subsection{Biodegradable ceramic materials}

Bioceramics can be divided into inert bioceramics, surface active bioceramics, absorbable bioceramics and composite ceramics composed of bioceramics and other materials. Biodegradable absorbable bioceramics mainly refer to materials that can be gradually degraded and absorbed in the biological tissue and can be replaced by the new tissue such as the calcium phosphate ceramic and calcium phosphate bone cement.

Calcium phosphate is a kind of ceramic material with good biological affinity which has similar mineral composition with human skeletal tissue, and it has good biocompatibility, safety, non-toxic, can be used as implant materials guiding the growth of new bone. 
The traditional calcium phosphate bone cement is a kind of crystal which is synthesized by Calcinated the mixture of calcium and phosphate under the temperature of $800 \mathrm{to} 01300^{\circ} \mathrm{C}$, which can not be absorbed in the body. While the calcium phosphate bone cement recently studied is a kind of non ceramic, various calcium phosphate directly crystallize in vivo, no heating is required and can be gradually absorbed and replaced by host bone in the body. The clinical application research mainly involves three areas: drug delivery carrier; auxiliary fixation for fracture treatment; as an artificial bone substitute material for bone defect.

\subsection{Biological derivative materials}

Biological derivative degradable materials are derived from special treatment of natural biological tissue, which can be divided into two categories: one is a mild process of fixing, sterilizing or eliminating antigenicity, maintaining the original organization configuration of the materials including catgut, freeze-dried bone, bovine carotid artery. The other one is strong process of breaking the original configuration and reconstruct new physical form such as coral artificial bone.

The crystal structure of coral artificial bone is mainly pure hydroxyapatite and contains trace amounts of beta calcium phosphate, which is the same as human bone tissue. The degradation of porous light based apatite is composed of two parts: the reabsorption of osteoblasts and chemical dissolution of itself. At present, coral and its derivatives have been widely used in skull surgery and orthopedics.

\subsection{Hybrid materials}

The hybrid of materials with biological activity and the general ones is also called as biologically active composite material. Such materials can stimulate and actively induce body's own repair and regeneration ability, so as to the lesion tissue and organ eventually completely or mainly replaced by the regenerated natural healthy tissue or organ.

One of representative for biologically active materials is bone morphogenetic protein (BMP). BMP can induce the differentiation of mesenchymal cells into the chondrocytes, osteoblasts and osteocytes, increasing the resource of osteoblasts and greatly speeding up the rate of osteogenesis. BMP biodegradable composite is a kind of new functional material with active inducing bone repair ability which combines BMP with some degradable carrier materials. The materials continue to dissolve out space for the growth of new bone when the surface BMP in the composite induct the formation of new bone and finally new bone entirely or mainly composed of natural bone is formed [8, 9].

\section{The application of degradable materials in medical field}

\subsection{Artificial bone material}

Artificial bone material can be divided into inorganic materials, organic materials and composite materials according to the structure and properties. According to resource, it can be divided into two categories, synthetic materials and natural materials. Currently commonly used artificial bone materials for clinical are biological ceramic inorganic materials, such as hydroxyapatite, mostly are prepared by high temperature sintering, the onlooke structure and physical chemical performances are far different from normal bone tissue, which is difficult to degrade after implanted, not conducive to the formation of new bone tissue in transplantation area. Therefore, artificial bone materials become the research focus $[10,11]$.

The commonly used material of biodegradable materials is polylactic acid. Polylactic acid is a kind of organic polymer material formed by lactic acid monomer polymerizing under certain condition. A large number of experiments showed that the material not only has good biological compatibility and bone conduction performance, but also can be completely biodegraded and the product is lactic acid, which will enter metabolism circulation of human body and eventually translate into carbon dioxide and water. Polylactic acid has good elastic modulus and thermal formability, we can control the mechanical properties of polylactic acid, the degradation speed by adjusting the molecular weight, and the choice of different polymerization molding method, meeting the different clinical requirements. 


\subsection{Tissue engineering}

Cell culture scaffold material is one of the important content in the research of tissue engineering, is the key to realize industrialization. At present, the developed biological materials for cell culture in tissue engineering scaffolds are mainly divided into two categories, namely synthetic materials and natural biological materials. Natural biological materials have the advantages of cellular signal identification, cell adhesion promoting, proliferation and differentiation, good biocompatibility, good biodegradability, showing incomparable advantages of the synthetic materials.

Natural biological materials used for cell culture are mainly divided into two categories: polysaccharide and protein. Polysaccharide mainly include chitin, hyaluronic acid and its derivatives, etc.; Proteins mainly include collagen protein and blood fibrin.

\subsection{Organ repair}

At present, biodegradable materials used in the repair of peripheral nerve injury include water gel, biofilm, chitin and chitosan, poly lactic acid, glycolic acid tubes to transplant, polylactic acid and copolymer, etc. Although the above biodegradable materials are one of the best way to solve the nerve defect, but the effect is different because of different composition and structure.

\section{Conclusion}

Biodegradable materials showed a broad application prospect because of good characteristics, having a great potential in the biomedical engineering. While as degradable biomedical materials is more and more widely used, the studies about biomedical materials is becoming more and more deep, it must also meet other conditions on the basic requirements of biocompatibility. Therefor, further research is needed. Biodegradation is one of the important properties of biomedical materials, it is important for the application of biomedical materials in the body. So now the main problem we are faced with is how to design a new material with more extensive use according to its mechanical properties and degradation rate $[1,3,4,11,13]$.

\section{References}

[1] Chu C C. Biodegradable Polymers: Volume 1: Advancement in biodegradation study and applications[J]. 2015.

[2] Fakirov S. 1. Biodegradable polyesters: synthesis, properties, applications[M]// Biodegradable Polyesters. Wiley-VCH Verlag GmbH \& Co. KGaA, 2015:1-19.

[3] Hou C L. Views on current medical biomaterials[J]. Chinese Journal of Bone \& Joint, 2015.

[4] Jin J. Research on the tpe biodegradable biomedical polymer materials[J]. 2015.

[5] Xu C, Yepez G, Wei Z, et al. Synthesis and characterization of conductive, biodegradable, elastomeric polyurethanes for biomedical applications[J]. Journal of Biomedical Materials Research Part A, 2016, 104(9):2305-2314.

[6] Vinicius M, Andr T, Lirio S, et al. Powder metallurgical processing of iron for biodegradable medical implants: Physical and mechanical[J]. Frontiers in Bioengineering \& Biotechnology, 2016, 4.

[7] Rafiee-Pour H A, Moradian M, Sayadi R. Synthesized Biodegradable Biomaterial in Medicine[C]// Iranian Congress on Progress in Tissue Engineering and Regenerative Medicine. 2015.

[8] Integran Technologies I. In-vivo biodegradable medical implant comprising a microstructure engineered metallic material[J]. 2016.

[9] G. Storti, M. Lattuada. 8 - Synthesis of bioresorbable polymers for medical applications[J]. Bioresorbable Polymers for Biomedical Applications, 2017:153-179. 
[10] Paramsothy M, Ramakrishna S. Biodegradable materials for clinical applications: A Review[J]. Reviews in Advanced Sciences \& Engineering, 2015, 4(3):221-238.

[11] Kaminskii I P, Lozhnikova A V, Fomin G G, et al. Biodegradable materials application: experience of the russian technological platform "medicine of the future” $[\mathrm{J}]$. Key Engineering Materials, 2016, 683:440-446.

[12] Khalil I, Irorere V, Radecka I, et al. Poly- $\gamma$-glutamic acid: niodegradable polymer for potential protection of beneficial viruses[J]. Materials, 2016, 9.

[13] Hou L D, Li Z, Pan Y, et al. A review on biodegradable materials for cardiovascular stent application[J]. Frontiers of Materials Science, 2016:1-22. 